\title{
Majorana Zero Modes in Graphene
}

\author{
P. San-Jose, ${ }^{1}$ J. L. Lado, ${ }^{2}$ R. Aguado, ${ }^{1}$ F. Guinea, ${ }^{3,4}$ and J. Fernández-Rossier ${ }^{2,5}$ \\ ${ }^{1}$ Instituto de Ciencia de Materiales de Madrid, Consejo Superior de Investigaciones Científicas (ICMM-CSIC), \\ Sor Juana Inés de la Cruz 3, 28049 Madrid, Spain \\ ${ }^{2}$ International Iberian Nanotechnology Laboratory (INL), Avenida Mestre José Veiga, \\ 4715-330 Braga, Portugal \\ ${ }^{3}$ Instituto Madrileño de Estudios Avanzados en Nanociencia (IMDEA-Nanociencia), 28049 Madrid, Spain \\ ${ }^{4}$ Department of Physics and Astronomy, University of Manchester, Manchester M13 9PL, United Kingdom \\ ${ }^{5}$ Departamento de Fisica Aplicada, Universidad de Alicante, 03690 Alicante, Spain
}

(Received 27 July 2015; published 15 December 2015)

\begin{abstract}
A clear demonstration of topological superconductivity (TS) and Majorana zero modes remains one of the major pending goals in the field of topological materials. One common strategy to generate TS is through the coupling of an $s$-wave superconductor to a helical half-metallic system. Numerous proposals for the latter have been put forward in the literature, most of them based on semiconductors or topological insulators with strong spin-orbit coupling. Here, we demonstrate an alternative approach for the creation of TS in graphene-superconductor junctions without the need for spin-orbit coupling. Our prediction stems from the helicity of graphene's zero-Landau-level edge states in the presence of interactions and from the possibility, experimentally demonstrated, of tuning their magnetic properties with in-plane magnetic fields. We show how canted antiferromagnetic ordering in the graphene bulk close to neutrality induces TS along the junction and gives rise to isolated, topologically protected Majorana bound states at either end. We also discuss possible strategies to detect their presence in graphene Josephson junctions through Fraunhofer pattern anomalies and Andreev spectroscopy. The latter, in particular, exhibits strong unambiguous signatures of the presence of the Majorana states in the form of universal zero-bias anomalies. Remarkable progress has recently been reported in the fabrication of the proposed type of junctions, which offers a promising outlook for Majorana physics in graphene systems.
\end{abstract}

DOI: 10.1103/PhysRevX.5.041042

\section{INTRODUCTION}

The realization of topological superconductivity (TS), a novel electronic phase characterized by Majorana excitations, has become a major goal in modern condensedmatter research. Despite promising experimental progress [1-13] on a number of appealing implementations [14-17], a conclusive proof of TS remains an open challenge. Here, we report on a new approach to obtain TS and Majorana states in graphene-superconductor junctions. Key to our proposal is the interaction-induced magnetic ordering of graphene's zero Landau level (ZLL). Coupling this unique state to a conventional superconductor gives rise to novel edge states whose properties depend on the type of magnetic order. In particular, the canted antiferromagnetic phase is a natural host for Majorana bound states. Our proposal combines effects that were recently demonstrated experimentally (tunable spin ordering of the ZLL $[18,19]$

Published by the American Physical Society under the terms of the Creative Commons Attribution 3.0 License. Further distribution of this work must maintain attribution to the author(s) and the published article's title, journal citation, and DOI.
Subject Areas: Graphene, Superconductivity, Topological Insulators and ballistic [20,21] graphene-superconductor junctions of high transparency [21] operating in the quantum Hall regime [20]) and is thus ready to be tested.

While intrinsic TS is rare, it can be synthesized effectively through the coupling of a conventional $s$-wave superconductor (SC) and tailored electronic gases with spin-momentum locking. Using this recipe, it has been predicted that Majorana excitations should emerge when one induces superconductivity onto topological insulators [14] or semiconductors with strong spin-orbit coupling [15]. Particularly attractive are implementations of onedimensional TS using either semiconducting nanowires $[16,17]$ or edge states in two-dimensional quantum spin Hall (QSH) insulators since the main ingredients are already in hand. These ideas have spurred a great deal of experimental activity [1-13]. Despite this progress, however, an unambiguous demonstration of TS is, arguably, still missing. Important limitations of these systems include disorder, bulk leakage, or the imperfect proximity effect (the so-called soft gap problem). Thus, it is worthwhile to explore alternative materials.

One particularly interesting option is graphene [22], which exhibits very large mobilities even in ambient 
conditions, and where a ballistic proximity effect has recently been demonstrated [21]. Graphene was the first material in which a topological insulating phase was proposed [23] in the presence of a finite intrinsic spinorbit coupling. Kane and Mele showed that a gap opens at the Dirac point, and a single helical edge mode with a spinlocked-to-propagation direction develops at each edge. In such a QSH regime, gapping the edge states through proximity to a conventional superconductor gives rise to a one-dimensional TS along the interface [14]. Graphene's negligible spin-orbit coupling, however, has proved to be a fundamental roadblock in this program.

In this work, we present a simple mechanism to realize the above situation in graphene without recourse to spinorbit coupling. We consider a graphene ribbon in the quantum Hall $(\mathrm{QH})$ regime, in which, unlike in the QSH case, time-reversal symmetry is broken by a strong magnetic flux (Appendix A). In contrast to conventional twodimensional electron gases, graphene develops a zero Landau level at the Dirac point, which has been shown in pristine samples to become split because of electronic interactions [24-29]. Experimental evidence [19] points towards spontaneous antiferromagnetic ordering [30-32], although other broken symmetries have been discussed [18]. In this work, we consider all possible magnetic orders. Figure 1 summarizes the different possibilities, ranging from ferromagnetic $(\mathrm{F})$ to antiferromagnetic $(\mathrm{AF})$ ordering [33], including canted AF which may be controlled by an external in-plane Zeeman field as argued in Ref. [19]. The different orders are parametrized by the angle $\theta$ between the spin orientation of the ZLL in the two graphene sublattices, so that $\theta=0$ for $\mathrm{F}$ and $\theta=\pi$ for $\mathrm{AF}$.

While $\theta$ is considered, in our model, as an externally tunable parameter as in Ref. [19], we have checked that a mean-field calculation in a honeycomb Hubbard model under an in-plane Zeeman field (Appendix A) yields the same bulk and edge phenomenology presented in this work [31]. Corrections beyond the mean field and the Hubbard model have been explored theoretically in the past and have predicted the formation of a Luttinger liquid domain on an infinite vacuum edge [34]. The corresponding excitation density resembles the noninteracting edge for $\mathrm{F}$ order, rather than the $\mathrm{AF}$ case. The interacting problem at a highly transparent superconducting contact remains an open problem. We conjecture that, given their robust topological origin, the Majorana phenomena described here at a meanfield level would survive in the Luttinger regime, at least within a limited range of parameters, and with power-law corrections to the transport results. These issues, however, remain beyond the scope of this work.

\section{TOPOLOGICAL SUPERCONDUCTIVITY IN QUANTUM HALL GRAPHENE}

An infinite ferromagnetically ordered $(\theta=0)$ ribbon in vacuum has a $\mathrm{QH}$ mean-field band structure [35] as shown
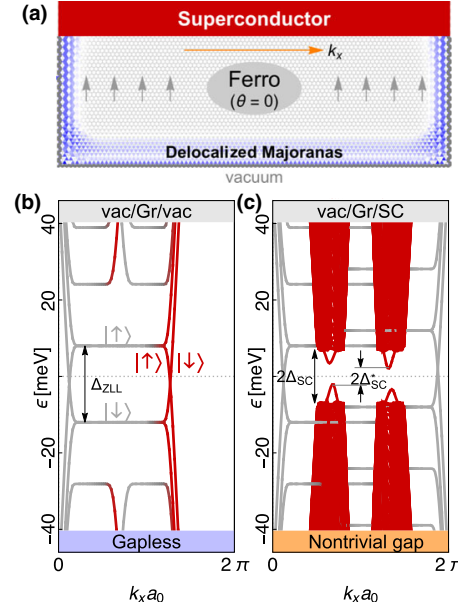
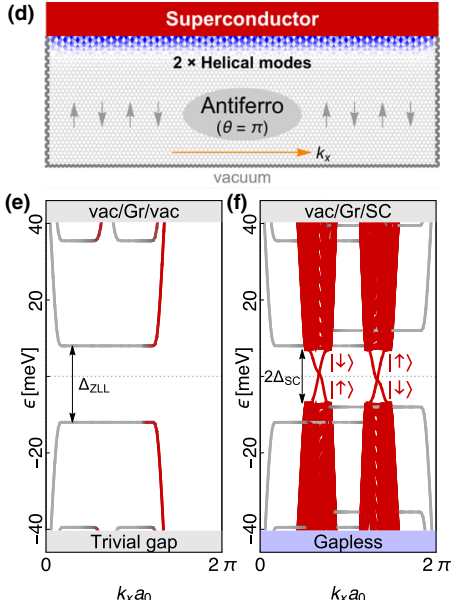
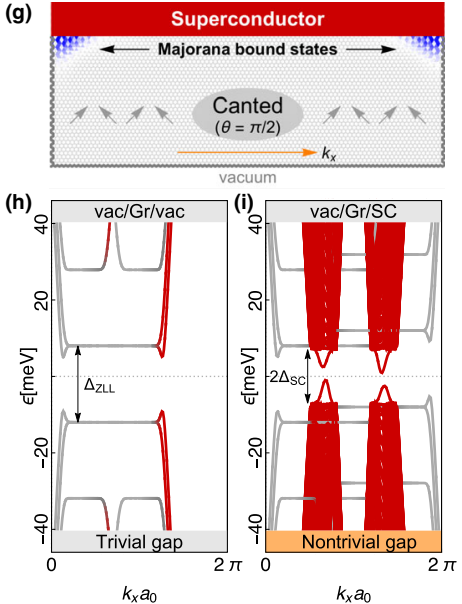
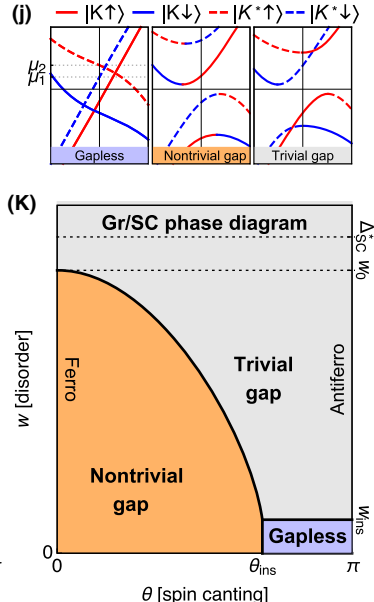

FIG. 1. Sketch, band structure, and phase diagram of a 350-nm-wide graphene sample (Fermi velocity $v_{F}=10^{6} \mathrm{~m} / \mathrm{s}$ ) in the quantum Hall regime (out-of-plane field $B_{z}=1 \mathrm{~T}$ ) in various configurations. The chemical potential (dotted line) is tuned within the gap $\Delta_{\mathrm{ZLL}} \approx 20 \mathrm{meV}$ of the zero Landau level, which has ferromagnetic (a-c), antiferromagnetic (d-f), or canted antiferromagnetic ordering $(\mathrm{g}-\mathrm{i})$ due to electronic interactions. The band structures under each sketch correspond to an infinite graphene ribbon surrounded by vacuum (vac/Gr/vac panels) or coupled to a superconductor of gap $\Delta_{\mathrm{SC}}$ (chosen to be large, around $7 \mathrm{meV}$, for visibility) along the top edge as in the sketches (vac/Gr/SC panels, Nambu bands). Bands in red correspond to eigenstates localized at the top edge of the ribbon. The zero-energy local density of states is shown in blue in each sketch. (j) Low-energy band structure of states along a graphenesuperconductor interface folded onto the $\Gamma$ point, in the gapless (left panel, zoom of panel f), nontrivially gapped (middle panel, canted order), and trivially gapped (right panel, with strong intervalley scattering) phases. (k) Phase diagram of said interface, computed from its low-energy effective Hamiltonian (see text), as a function of magnetic angle $\theta$ and intervalley coupling $w$. The three possible phases are shown, bounded by threshold values $w_{0}, w_{\text {ins }}$, and $\theta_{\text {ins }}$ (see main text). 
in Fig. 1(b). (Details on the modeling are given in Appendix A). The ZLL is spin split into the two spin sectors in the direction of the ferromagnetic order, denoted by $|\uparrow\rangle,|\downarrow\rangle$ at energies $\pm \Delta_{\text {ZLL }} / 2$ with respect to the Dirac point. For energies within this gap, a single pair of gapless counterpropagating spin-polarized edge states develop, shown in red for states at the upper vacuum edge of the sample. Note the peculiar situation created in this energy window: Edge states are not chiral-like in the conventional QH regime but may rather propagate in both directions with opposite spins, like in the QSH regime. Also, valley degeneracy is lifted at any given edge, which hosts a single state per propagating direction. Upon contacting one edge to a conventional superconductor of gap $\Delta_{\mathrm{SC}}$ [as in the sketch of Fig. 1(a)], while keeping the chemical potential (dotted line) within the ZLL gap, the edge states along the interface develop an induced gap $\Delta_{\mathrm{SC}}^{*}$ [see the corresponding Nambu band structure of Fig. 1(c) - the red lines, once more, indicate states localized at the upper edge of the graphene ribbon, now including the dense quasiparticle spectrum of the superconductor]. The gap $\Delta_{\mathrm{SC}}^{*}$ is an important scale in this problem since it turns out to be a topologically nontrivial gap. This is confirmed by computing the band structure's $\mathbb{Z}_{2}$ topological invariant, Eq. (B2), relevant for quasi-one-dimensional $D$-class systems [36]. Unlike in a conventional QSH system, where time-reversal symmetry is required, the vacuum edge states do not immediately develop a topological gap when contacted to the supercoductor. This only happens with a sufficiently good contact. On the other hand, while the conventional QSH metal becomes destroyed by any time-reversalbreaking perturbation (such as inelastic scattering or magnetic impurities), which in turn spoil the nontrivial superconducting gap, this is not the case of the present implementation, which has a broken time-reversal symmetry from the start. Moreover, we emphasize once more that no spin-orbit coupling at all is necessary for $\Delta_{\mathrm{SC}}^{*}$ to develop.

The immediate consequence of a nontrivial gap topology is the appearance of zero-energy Majorana bound states (MBSs) at an interface with a trivial insulator, following the bulk-boundary correspondence principle. In this case, however, both ends of the topologically gapped superconducting interface are coupled to a gapless vacuum edge so that the zero modes become delocalized into the continuum away from the interface [see the zero-energy local density of states (LDOS) in blue in Fig. 1(a)]. This situation is similar to the fate of MBSs at the ends of topological proximized semiconductor nanowires when strongly coupled to a metallic environment [37].

The electronic structure associated with an antiferromagnetic ribbon $[\theta=\pi$, Fig. $1(\mathrm{~d})]$ is the opposite. The states along a vacuum edge are now (trivially) gapped [31] like the ZLL itself [Fig. 1(e)]. Surprisingly, when contacting the edge to a conventional superconductor, two pairs of gapless helical edge modes emerge with spin-momentum locking around conjugate momenta $K$ and $K^{*}$ [Fig. 1(f)]. These unexpected states, spatially spread along the interface [see the blue LDOS in Fig. 1(d)], are decoupled electron-hole $(e-h)$ superpositions with orthogonal and well-defined spin orientation along the AF axis, $\left|K^{(*)} \uparrow\right\rangle=$ $a\left|\phi_{e \uparrow}\right\rangle+b\left|\phi_{h \downarrow}\right\rangle$ and $\left|K^{(*)} \downarrow\right\rangle=a^{\prime}\left|\phi_{e \downarrow}\right\rangle+b^{\prime}\left|\phi_{h \uparrow}\right\rangle$. A full discussion of these states is presented in Appendix D. The two helical edge modes remain gapless as long as no AF canting is present in graphene $(\theta=\pi)$ and intervalley scattering is zero at the interface. We next consider deviations from these two assumptions.

Canting of the AF order may be induced by means of a large enough in-plane Zeeman field and is thus, to some extent, externally tunable. This idea was employed in Ref. [19] to tune a graphene QH bar in vacuum between the $\mathrm{AF}$ and $\mathrm{F}$ regimes, leading to an insulator-to-helical metal transition in edge transport [evolution from Fig. 1(e) to 1(b)]. A typical canted AF band structure $(\theta=\pi / 2)$ is shown in Fig. 1(h). The vacuum edge states exhibit a topologically trivial and $\theta$-dependent gap, smaller than the bulk $\Delta_{\text {ZLL}}$. Along a superconductor interface, the canted AF helical states are also gapped [Fig. 1(i)]. Like in the ferromagnetic case, this gap is topologically nontrivial. This situation allows for the emergence of true localized zero-energy MBSs at the ends of the superconductor interface, where the edge gap changes topology [see Fig. 1(g)]. The MBSs are topologically protected and are not destroyed by any small perturbations or even by modifying the crystal structure of the superconductor (Appendix E).

To understand the full phase diagram of the graphenesuperconductor interface quantitatively, it is useful to employ a simplified description in terms of an effective low-energy Hamiltonian for the edge states (see Appendix B). The model is valid for a chemical potential tuned to the ZLL gap and has the advantage of allowing us to incorporate the effects of atomic disorder along the junction (encoded in an intervalley coupling $w$, where "valley" here refers to the conjugate $K$ and $K^{*}$ momenta) and arbitrary spin canting (encoded in an intravalley splitting $b_{\theta}=\Delta_{\mathrm{SC}}^{*} \cos [\theta / 2]$ ). It correctly describes the three possible phases for low-energy interface modes: gapless, trivially gapped, and nontrivially gapped. The corresponding phase diagram is shown in Fig. 1(k). Typical edgemode dispersions within each phase (with $K$ and $K^{*}$ points folded onto the $\Gamma$ point) are shown in Fig. 1(j) and are characterized by their energies $\mu_{1,2}<\Delta_{\mathrm{SC}}^{*}$ at $k_{x}=0$ for $\theta=\pi[\mathrm{AF}], w=0$, and their corresponding velocities $v_{1,2}>0$ (left panel).

The gapless interface regime [light blue in Fig. 1(k)] is achieved for $|w|<w_{\text {ins }} \equiv \frac{1}{2}\left|\mu_{1} \sqrt{v_{2} / v_{1}}-\mu_{2} \sqrt{v_{1} / v_{2}}\right|$ and $\quad \theta>\theta_{\text {ins }}$, where $\quad b_{\theta_{\text {ins }}}=\Delta_{\mathrm{SC}}^{*} \cos \left(\theta_{\text {ins }} / 2\right) \equiv$ $\frac{1}{2}\left|\mu_{1} \sqrt{v_{2} / v_{1}}+\mu_{2} \sqrt{v_{1} / v_{2}}\right|$. The gapped regimes are characterized by the $\mathbb{Z}_{2}$ topological invariant of the system, 
which reads $\nu=\operatorname{sign}\left(w^{2}+\mu_{1} \mu_{2}-b_{\theta}^{2}\right)$ (see Appendix B). A trivially gapped phase $\nu=+1$ is reached for strong intervalley coupling $w$ at the interface, while for intervalley scattering below a threshold $w<\sqrt{b_{\theta}^{2}-\mu_{1} \mu_{2}}$, the interface is one-dimensional TS with invariant $\nu=-1$. The nontrivially gapped regime is most robust against disorder for $\mathrm{F}$ order, for which the threshold $w$ reaches its maximum $w_{0}=\sqrt{\left(\Delta_{\mathrm{SC}}^{*}\right)^{2}-\mu_{1} \mu_{2}}$. Note that to achieve a nontrivial TS interface, the intervalley coupling $w$ should therefore never exceed the induced gap $\Delta_{\mathrm{SC}}^{*}$ (this is always the case for sufficiently transparent junctions).

\section{EXPERIMENTAL SIGNATURES OF GRAPHENE MAJORANAS}

We finally consider measurable signatures of the MBSs in the system. A powerful probe whose feasibility has recently been demonstrated experimentally [20,21] involves interferometry of critical currents in Josephson junctions, and Fraunhofer pattern anomalies in particular [9,12,38]. Lee et al. predicted [39] that topological superconductivity in a short and wide Josephson junction could be directly detected in its Fraunhofer pattern, in the form of nonvanishing minima of the critical current for arbitrary magnetic flux through the junction. Such a Fraunhofer anomaly was recently observed in a three-dimensional topological insulator and was interpreted as possible evidence of MBSs [12].

Figure 2 shows the Fraunhofer pattern in a short and wide $(10-\mathrm{nm} \times 3-\mu \mathrm{m})$ graphene Josephson junction in various regimes. The black (bottom) curve corresponds to the noninteracting case (no magnetic ordering, $\left.\Delta_{\text {ZLL }}=0\right)$, which exhibits the conventional $I_{c}(\Phi)=$ $I_{c}^{0}\left|\sin \left(\pi \Phi / \Phi_{0}\right)\right| /\left(\pi \Phi / \Phi_{0}\right)$ critical current that decays as the inverse magnetic flux $\Phi$ through the junction $I_{c} \sim 1 / \Phi$ and vanishes at multiples of the flux quantum $\Phi_{0}=h / 2 e$. A similar behavior is obtained in the gapless regime $\theta>\theta_{\text {ins }}, w<w_{\text {ins }}$ (purple curve, with $\theta=\pi[\mathrm{AF}]$ and $w=0$ ). In both cases, the junction is host to a narrow quasicontinuum of Andreev bound states around the Fermi energy for any value of the superconducting phase difference $\phi$. The corresponding spectra are shown in the top row of inset (a) (for $\Phi=15.5 \Phi_{0}$ ). States in red and blue are localized at the top and bottom edges of the junction [inset (b)], respectively, with gray denoting states spread across the junction [inset (c)]. The case with trivially gapped interfaces (strong intervalley scattering) also exhibits a generic Fraunhofer pattern with vanishing minima (light gray curve, $\theta=0[\mathrm{~F}]$ and $w>w_{0}$ ). The minima, however, occur at fluxes that are shifted away from integer $\Phi / \Phi_{0}$ at high $\Phi$, while the maxima do not decay like the conventional $I_{c} \sim 1 / \Phi$ pattern, which is connected to nonuniform currents across the junction [38]. The Andreev spectrum of the trivially gapped phase is qualitatively different from the gapless spectra and generally shows a distinct gap devoid of

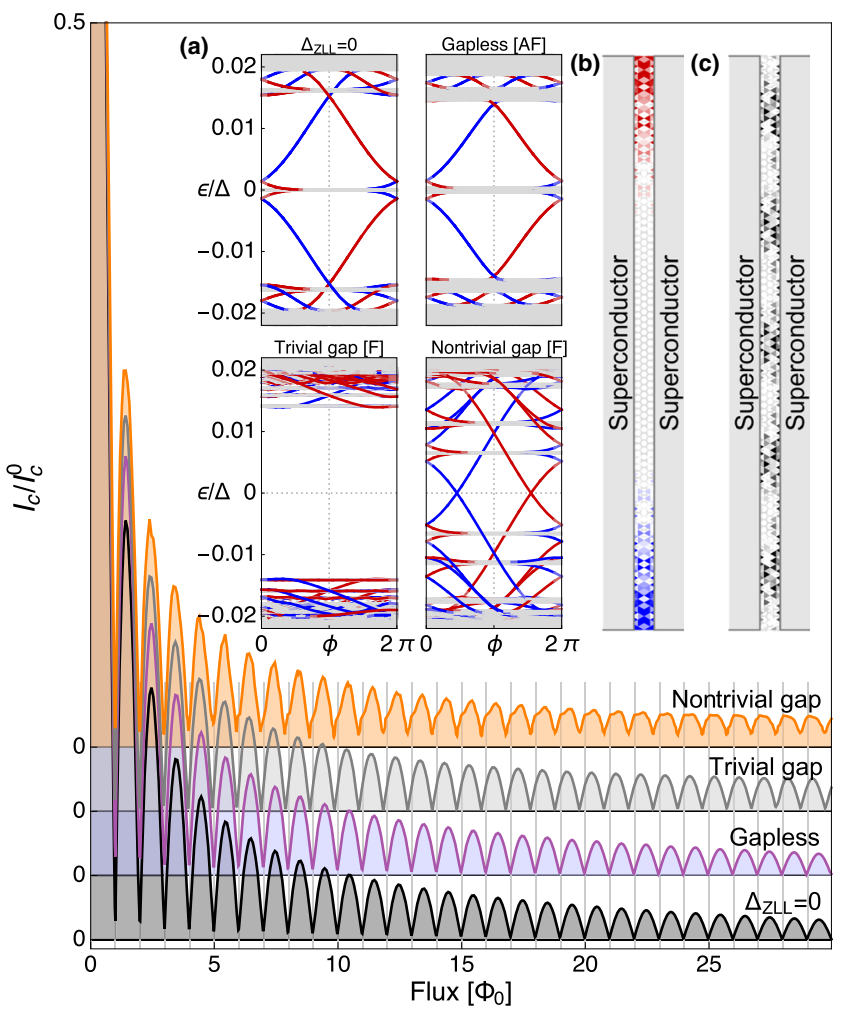

FIG. 2. Normalized critical current $I_{c} / I_{c}^{0}$ as a function of magnetic flux through a Josephson junction. Curves from bottom to top (shifted for better visibility) correspond to the noninteracting case (black), the gapless AF phase (purple), a trivially gapped AF phase (light gray), and a ferromagnetic phase with a topologically nontrivial gap (orange). Only the latter shows nondecaying nonzero minima in $I_{c}$, a consequence of an Andreev spectrum [inset (a)] with an odd number (one) of edge-resolved zero-energy crossings (bottom-right panel). States colored in red and blue are located at the top and bottom edges, respectively [inset (b)], while states in gray are spread across the width of the junction [inset (c)].

any edge states [inset (a), bottom left]. For certain values of parameters, it may exhibit zero-energy crossings inside the gap, but in such cases, these crossings are accidental (not topologically protected) and there is always an even number of them at a given edge.

The Josephson junction with a nontrivial gap along the contacts is distinctly different from all previous cases. This phase develops two MBSs at each edge (top and bottom), which hybridize to carry a finite supercurrent that never vanishes as the flux increases. The corresponding Fraunhofer pattern thus exhibits a finite background with a superimposed nondecaying oscillation (orange curve in Fig. 2), as described in Ref. [39]. The finite minima are roughly one-half of the maxima at large flux and occur away from integer $\Phi / \Phi_{0}$, at values very close to the zeros of the trivially gapped phase. (Note, however, that such a distinctive pattern develops only in junctions shorter than the Majorana localization length and pierced by a large 
number of flux quanta, so the Majoranas are well developed and opposite edges are decoupled). These Fraunhofer anomalies, although not completely unambiguous, thus constitute a measurable hint of the presence of MBSs at the end of a graphene-superconductor interface. Unfortunately, fabricating a very short junction is challenging, in particular, because of charge-transfer effects from the superconductors which were neglected here and which will dope graphene away from neutrality within a few nanometers of the contacts. It is thus important to explore other less stringent experimental schemes that are, at the same time, not ambiguous. The key is to probe the Andreev spectrum directly for signatures of Majoranas and nontrivial topology.

The presence of the two hybridized Majoranas per vacuum edge in the nontrivial phase manifests in the Andreev spectrum as a single, topologically protected, zero-energy crossing at each edge as $\phi$ is increased by $2 \pi$ [one red and one blue crossing; see bottom-right inset (a) in Fig. 2]. An odd number of such zero-energy crossings has been shown [40] to be a direct manifestation of nontrivial topological order $\nu=-1$ and is the underlying reason for the anomalous Fraunhofer pattern of the junction. A completely nonambiguous demonstration of the presence of MBSs is thus also possible, in principle, by directly counting edge-resolved zero-energy crossings using Andreev spectroscopy [41] in a phase-controlled Josephson junction. This may be achieved by measuring differential conductance $d I / d V$ through a normal point contact attached to one edge of the junction, as sketched in Fig. 3(a). We assume a single spinful channel is open. Each Andreev level of energy $\epsilon$ in the junction is detected as a $d I / d V$ resonance through the probe at bias $V=\epsilon / e$, with a resonance width that measures the state's probability density at the point contact. Figures 3(b) and 3(d) show a simulation (see Appendix $\mathrm{C}$ for details) of such a $d I / d V$ as a function of $\phi$ (at $\theta=0$ and $\theta=0.1 \pi$, respectively) for a square $500-\mathrm{nm} \times 500-\mathrm{nm}$ Josephson junction. Note that, unlike in the Fraunhofer simulation, this is not a short junction since that is no longer a desirable or realistic requirement in the context of Andreev spectroscopy. The number of edge-resolved zero-energy crossings as $\phi$ is swept from 0 to $2 \pi$ is one, as corresponds to Majoranahosting SC contacts [compare this $d I / d V(\phi)$ to the blue lines in the bottom-right inset, Fig. 2(a) (short junction)]. Incidentally, the $d I / d V(\phi)$ profiles at zero (nonzero) $\theta$ in panel (b) [(d)] follow the characteristic Andreev-level spectra in topological Josephson junctions through long semiconducting nanowires at perfect (nonperfect) transparency [42-46]. The conductance at the crossings is pinned to a universal value $4 e^{2} / h$ (white spot) (see Appendix C).

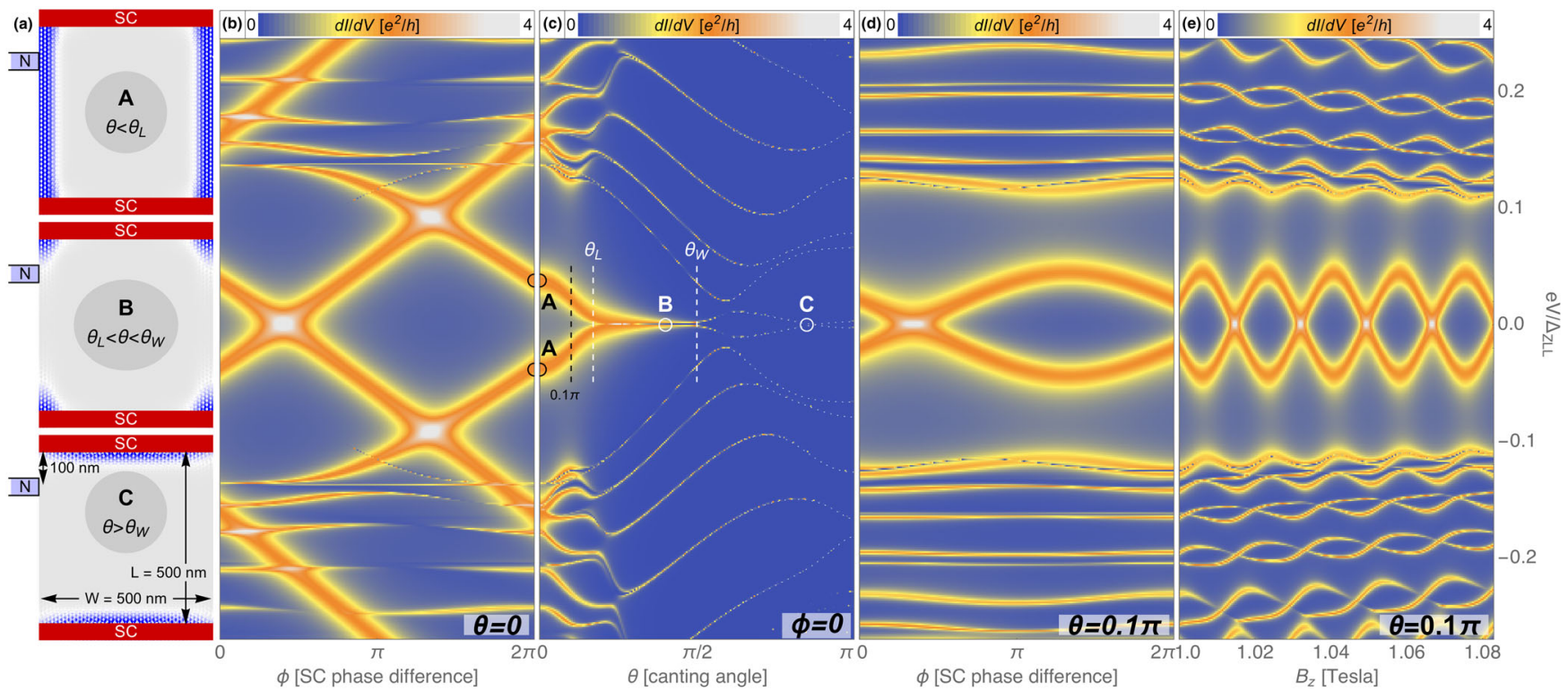

FIG. 3. (a) In blue, density of the lowest Andreev level formed by the hybridization of four Majorana bound states in a square 500-nm $\times 500-n m$ Josephson junction at different values of canting angle $\theta$. The rest of the parameters are as in Fig. 1 . In A and $\mathrm{C}$ $\left(\theta<\theta_{L}\right.$ and $\theta>\theta_{W}$, respectively), the hybridization is strong, while in $\mathrm{B}$, it is exponentially suppressed, and the level remains a fourfold degenerate zero mode. (b-e) Transport spectroscopy $d I / d V$ from a normal point contact $N$ (transparent, single spinful channel) [see (a)]. (b, d) The $d I / d V$ (at $\theta=0$ and $\theta=0.1 \pi$, respectively) as a function of the junction phase difference $\phi$. Both exhibit an odd number (one) of zero-energy crossings of edge-resolved $d I / d V$ resonances, signaling a topologically nontrivial system. (c) $d I / d V$ as a function of canting angle $\theta$ at $\phi=0$. It exhibits a zero-bias anomaly in the window $\theta_{L}<\theta<\theta_{W}$ (see dashed lines). (e) The $d I / d V$ dependence with out-of-plane magnetic field $B_{z}$ through the sample $(\phi=0, \theta=0.1 \pi)$, showing the same zero crossings as with $\phi$ each time the total flux $\Phi=B_{z} L W$ is increased by a flux quantum $\Phi_{0}$. 
While a $\phi$-controlled junction typically requires a SQUID-like geometry and may be experimentally challenging, the existence of MBSs in the junction may be detected even more simply by varying the out-of-plane magnetic field $B_{z}$ and hence the total flux $\Phi$ through graphene. An increase of $\Phi$ by $\Phi_{0}$ is equivalent to increasing $\phi$ by $2 \pi$ for large $\Phi / \Phi_{0}$. This is shown in Fig. 3(e) [compare to Fig. 3(d)]. Moreover, at fixed $B_{z}$ and $\phi=0$, the MBSs also show up as a zero-bias $d I / d V$ peak [Fig. 3(c)] as $\theta$ is tuned-by the in-plane Zeeman field $B_{\|}$- within a range $\theta_{L}<\theta<\theta_{\mathrm{W}} \leq \theta_{\text {ins }}$ (dashed lines). For the realistic parameters used in Fig. $3\left(B_{z}=1 \mathrm{~T}\right.$, $L=W=500 \mathrm{~nm}$ ), and using $B_{0}=10 \mathrm{~T}$ as the typical inplane field $B_{\|}$for complete Ferro polarization, $\theta_{L} \approx 0.17 \pi$ and $\theta_{W} \approx 0.5 \pi$ are reached for $B_{\|} \approx 9.6 \mathrm{~T}$ and $B_{\|} \approx 7.1 \mathrm{~T}$, respectively (see Appendix $C$ ). Inside this window [point $B$ in Figs. 3(a) and 3(c)], the four Majoranas are concentrated at the corners of the junction and do not overlap, as they decay within a distance smaller than both the width $W$ and length $L$ of the junction, and hence appear as a sharp zerobias resonance in the $d I / d V$ with exponentially small splitting. The resonance has a universal magnitude of $2 e^{2} / h$ at low temperatures (see Appendix C). This is analogous to the zero-bias anomalies reported in pioneering Majorana experiments on semiconducting nanowires [1]. In contrast, for $\theta<\theta_{L}$ (point $\mathrm{A}$, bulk approaching ferroordering), Majorana pairs overlap along the vacuum edge and produce a split resonance. Likewise for $\theta>\theta_{W}$ (e.g., point $\mathrm{C}, \mathrm{SC}$ contact close or inside the gapless regime), Majoranas overlap along the SC contact and develop a (roughly $\phi$-independent) splitting, making the $\theta>\theta_{L}$ junction strictly trivial. Note also the strong suppression of the width in the $d I / d V$ resonances in this case, due to the exponentially small wave-function amplitude at the point contact in this geometry (dotted lines overlaid for $\theta>0.6 \pi$ to improve visibility). Essentially the same $d I / d V(\theta)$ phenomenology is obtained in a setup with a single superconducting contact (which hosts two Majoranas instead of four) (see Appendix C).

\section{DISCUSSION}

Our results show that the spontaneous magnetic ordering of the ZLL in graphene enables the creation of topological superconductivity and Majorana states at an interface with a conventional superconductor, even in the absence of spin-orbit coupling in the system. The key is to tune the Fermi energy in the contact into the ZLL gap and to achieve a good proximity effect therein. The recently characterized samples of Ref. [20] are good candidates to realize our proposal. Impressive progress in controlling graphene filling into proximity gaps has also been reported [47]. We furthermore showed that nonvanishing and nondecaying supercurrent minima in the Fraunhofer pattern across a depleted graphene Josephson junction constitute a characteristic signal of topological order and the presence of Majorana bound states in the junction [39]. Fraunhofer patterns of extraordinary quality have recently been reported in high-transparency ballistic graphene Josephson junctions [21]. We predict even stronger observable signatures of nontrivial topology in Andreev transport spectroscopy, both in the form of an odd number of $4 e^{2} / h$ edge-resolved zero-bias crossings versus junction phase difference $\phi$ or out-of-plane magnetic field $B$, and extended $2 e^{2} / h$ zero-bias anomalies versus canting angle $\theta$. These experimental probes and the required device parameters are within reach in top laboratories today. We thus hope that the possibility of tuning graphene-superconducting interfaces into a topological phase hosting Majorana bound states could be tested soon.

\section{ACKNOWLEDGMENTS}

P. S.-J. and R. A. are grateful to A. Cortijo and L. Brey for stimulating discussions. We acknowledge the support of the European Research Council (F. G.), the Spanish Ministry of Economy and Innovation through Grants No. FIS201123713 (F. G. and P. S.-J.) and No. FIS2012-33521 (R. A.), the Ramón y Cajal Programme (P. S.-J.), the Marie-CurieITN 607904-SPINOGRAPH (J. L., J. F. R., and F. G.), and Generalitat Valenciana (ACOMP/2010/070), Prometeo (J. F. R.).

\section{APPENDIX A: MODELING}

In this section, we present the system models employed in this work. Noninteracting graphene can be modeled by a nearest-neighbour tight-binding Hamiltonian in an honeycomb lattice, with lattice constant $a_{0}$,

$$
H_{0}=-\sum_{\langle i, j\rangle, s} t e^{i \phi_{i j}} c_{j s}^{\dagger} c_{i s}+\sum_{i s} \mu_{N} n_{i s},
$$

where $n_{i s}=c_{i s}^{\dagger} c_{i s}, i$ is the site index, $s$ is the spin, and $\phi_{i j}=-(e / \hbar) \int_{\vec{r}_{i}}^{\vec{r}_{j}} d \vec{r} \cdot \vec{A}(\vec{r})$ is the Peierls phase due to the magnetic flux $\mathcal{B}_{z}=\hat{z} \cdot(\vec{\nabla} \times \vec{A})$. We consider a perturbation $\sum_{i} \vec{B} \cdot \vec{S}_{i}$ arising from an external Zeeman field $\vec{B}$, where $\vec{S}_{i}=\sum_{s s^{\prime}} c_{i s}^{\dagger} \vec{\sigma}_{s s^{\prime}} c_{i s^{\prime}}$ is the spin at site $i$. Furthermore, intrinsic electron-electron interactions are included in the local Hartree-Fock approximation [31], which then take the form of a self-consistent Zeeman-like field $\vec{B}_{U}\left(\vec{r}_{i}\right)$ that is different in the two honeycomb sublattices. The total Zeeman-like perturbation $H_{Z}$ thus reads

$$
H_{Z}=\sum_{i}\left[\vec{B}+\vec{B}_{U}\left(\vec{r}_{i}\right)\right] \cdot \vec{S}_{i}
$$

While $\vec{B}$ is uniform, favoring ferromagnetic (F) ordering, the self-consistent $\vec{B}_{U}\left(\vec{r}_{i}\right)$ is generally opposite for nearest 
neighbors, favoring antiferromagnetic ordering of the bulk. The combination of the two leads to a $\vec{B}$-tunable, spinordered ZLL that can be tuned from AF to F, as discussed in the main text. Further details on the mean-field numerics and results can be found in Ref. [31].

The hybrid graphene-superconductor (SC) system is described by the Hamiltonian

$$
H=H_{0}+H_{Z}+\sum_{\vec{r}_{i} \in \mathrm{SC}}\left[\Delta_{\mathrm{SC}} c_{i \uparrow}^{\dagger} c_{i \downarrow}^{\dagger}+\text { H.c. }\right] .
$$

The Fermi energy in $H_{0}$ above is $\mu_{N}$ for $\vec{r}_{i} \notin \mathrm{SC}$ in graphene and $\mu_{S}$ for $\vec{r}_{i} \in \mathrm{SC}$ at the superconductor [also a honeycomb lattice here, although this is not essential (see Appendix E)]. Similarly, $B, \vec{B}_{U}$, and $\mathcal{B}_{z}$ are zero in the superconductor. However, gauge invariance demands that for a finite magnetic flux in graphene, $\Delta_{\mathrm{SC}}(\vec{r})=$ $\Delta_{\mathrm{SC}} \exp \left[-(2 e / \hbar) \int^{\vec{r}} d \vec{r} \cdot \vec{A}(\vec{r})\right]$, where $\Delta_{\mathrm{SC}}$ is the pairing for zero flux at a given superconductor.

The critical currents for the Fraunhofer patterns have been calculated in a wide and short graphene Josephson junction, described by a discretized $H$ using an upscaled $a_{0}$ for numerical efficiency, following the ideas of Ref. [48]. The critical current for each magnetic flux is calculated as $I_{c}=2 e / \hbar \times \max _{\phi}(d F / d \phi)$, where $\phi$ is the superconducting phase difference and $F(\phi)$ is the free energy of the junction [49]. Exact diagonalization of the Hamiltonian is used to evaluate $F(\phi)$ at zero temperature. The method to compute the differential conductance for transport spectroscopy is explained in Appendix C.

\section{APPENDIX B: LOW-ENERGY DESCRIPTION OF A GRAPHENE-SUPERCONDUCTOR JUNCTION}

In this appendix, we derive a simplified effective Hamiltonian $H_{\text {eff }}$ for the two helical edge modes below the superconducting gap $\Delta_{\mathrm{SC}}$ that arise in a generic quantum Hall graphene ribbon and a superconductor. We also characterize its topology by deriving expressions for the relevant topological invariant as a function of model parameters.

We assume the chemical potential lies within the gap $\Delta_{\mathrm{ZLL}}$ induced by interactions in the zero Landau level (ZLL). The gap is associated with a bulk spin ordering described by a canting angle $\theta$ between the two sublattices. The effective model incorporates an arbitrary value for $\theta$ in graphene and also intervalley coupling due to atomic disorder along the interface. Formally, $H_{\text {eff }}$ is a projection of the microscopic Hamiltonian on the basis $\left\{|K \uparrow\rangle,|K \downarrow\rangle,\left|K^{*} \uparrow\right\rangle,\left|K^{*} \downarrow\right\rangle\right\}$ of the four AF helical edge states along the junction [see Fig. 1(f) in the main text, and their analytical description in the preceding section]. Furthermore, we linearize their dispersion in the AF case around $K$ and $K^{*}$ "valleys." These two valleys are folded onto the $\Gamma$ point by appropriately expanding the ribbon unit cell. Such folding allows us to include intervalley scattering into $H_{\text {eff }}$ in a simple way. $H_{\text {eff }}$ then takes the form $(\hbar=1)$

$H_{\mathrm{eff}} \approx\left(\begin{array}{cccc}\mu_{1}+v_{1} k_{x} & b_{\theta} & w & 0 \\ b_{\theta} & \mu_{2}-v_{2} k_{x} & 0 & w \\ w & 0 & -\mu_{2}-v_{2} k_{x} & b_{\theta} \\ 0 & w & b_{\theta} & -\mu_{1}+v_{1} k_{x}\end{array}\right)$.

Here, the intravalley coupling $b_{\theta}=\Delta_{S C}^{*} \cos (\theta / 2)$ implements AF canting and couples opposite spins within the same valley. The intervalley coupling $w$ is spin independent and corresponds to the harmonic of wave number $\Delta K=$ $\left(\vec{K}^{*}-\vec{K}\right) \cdot \hat{x}$ of any disorder term $W$ close to the interface, $w=\left\langle\phi_{\uparrow K^{*}}|W(\Delta K)| \phi_{\uparrow K}\right\rangle$. Both $b_{\theta}$ and $w$ can be chosen to be real without loss of generality. Here, $v_{1,2}>0$ are the velocities of the counterpropagating helical states, and $\mu_{1,2}<\Delta_{\mathrm{SC}}^{*}$ are their energies, relative to the Fermi energy, at the $\Gamma$ point [see Fig. $1(\mathrm{j})$ in the main text]. The overall structure apparent in $H_{\text {eff }}$ is fully determined by the particle-hole symmetry of the underlying Nambu description. Note that $H_{\text {eff }}$ only retains terms linear in momentum $k_{x}$ along the interface.

The simplicity of the linearized low-energy model above allows us to compute analytical expressions for the topological invariant and the boundaries that separate different phases of the junction (gapless, trivially gapped, nontrivially gapped), as summarized in the main text. We now briefly sketch their derivation.

\section{Topological invariant of edge Hamiltonian}

In symmetry class D (superconductors without timereversal symmetry), the one-dimensional topological invariant is $\mathbb{Z}_{2}$. It is conventionally defined, for periodic systems, as [50]

$$
\nu=\operatorname{sign}\left(\frac{\operatorname{Pf}\left[H(0) \tau_{x}\right]}{\operatorname{Pf}\left[H(\pi) \tau_{x}\right]}\right)=\frac{s_{0}}{s_{\pi}},
$$

where $s_{\alpha}=\operatorname{signPf}\left[H(\alpha) \tau_{x}\right], H\left(k_{x} a_{0}\right)$ is the 1D Bloch Hamiltonian for momentum $k_{x}, a_{0}$ is the lattice constant of the ribbon lattice, $\tau_{x}$ is the first Pauli matrix in the electron-hole sector, and Pf is the Pfaffian. It can be shown, in general, that $H \tau_{x}$ is antisymmetric at the high-symmetry points $k_{x} a_{0}=0, \pi$. The invariant $\nu$ is thus fully determined by the structure of $H$ at these two points. The effective Hamiltonian $H_{\text {eff }}$, Eq. (B1), only gives a faithful representation of the full microscopic Hamiltonian $H$ around one of them, the folded $\Gamma$ point $k_{x}=0$ [Fig. 1(j), main text]. To extract analytic results for $\nu$ for the full $H$ using $H_{\text {eff }}$, we must ensure that at the $\pi$ point, $s_{\pi}$ does not change when sweeping the parameter space (since this sector of states is not described by $H_{\text {eff }}$ ). This is indeed the case in our system 
for the chosen basis, for which $s_{\pi}=1$. The changes in topology stem from the reconnections of the low-energy edge states that are concentrated around $\Gamma$ and are well described by $H_{\text {eff }}$. Higher excited states not included in $H_{\text {eff }}$ never cross zero energy and therefore cannot affect the sign of the Pfaffian of $H(0) \tau_{x}$. One can thus write

$$
\nu=\operatorname{sign} \operatorname{Pf}\left[H_{\text {eff }}(0) \tau_{x}\right]=\operatorname{sign}\left(w^{2}+\mu_{1} \mu_{2}-b^{2}\right) .
$$

We have numerically verified the above result by evaluating the $\mathbb{Z}_{2}$ invariant exactly from the microscopic Hamiltonian $H$.

We finally sketch the derivation of the insulating thresholds $w_{\text {ins }}$ and $\theta_{\text {ins. }}$. These are extracted by computing the solutions for the wave number $k_{x}$ of $H_{\text {eff }}$ modes at zero energy. Since $H_{\text {eff }}\left(k_{x}\right)$ is linear in $k_{x}$, said $k_{x}$ solutions at $\epsilon=0$ can be obtained as eigenvalues of a matrix $-\left[\partial_{k_{x}} H_{\text {eff }}(0)\right]^{-1} H_{\text {eff }}(0)$. These can be worked out analytically and turn out to be all complex [i.e., the interface becomes gapped; see, e.g., Fig. 7(f)] if $w>w_{\text {ins }}$ or $\theta<\theta_{\text {ins }}$, with the expressions given in the main text,

$$
\begin{aligned}
w_{\text {ins }} & =\frac{1}{2}\left|\mu_{1} \sqrt{v_{2} / v_{1}}-\mu_{2} \sqrt{v_{1} / v_{2}}\right|, \\
b_{\theta_{\text {ins }}} & =\Delta_{\mathrm{SC}}^{*} \cos \left(\theta_{\text {ins }} / 2\right) \\
& \equiv \frac{1}{2}\left|\mu_{1} \sqrt{v_{2} / v_{1}}+\mu_{2} \sqrt{v_{1} / v_{2}}\right| .
\end{aligned}
$$

\section{APPENDIX C: TRANSPORT SPECTROSCOPY}

\section{Computing differential conductance}

The computation of the transport-spectroscopy results shown in Fig. 3 follows standard techniques of quantum transport. All interactions are incorporated into the meanfield solution for $\vec{B}_{U}$, Eq. (A2). In this case, the differential conductance $d I / d V$ through a normal contact with a single spinful channel at a certain bias $V$ is given by the BlonderTinkham-Klapwijk (BTK) formula $d I / d V=\left(2-R_{e e}+\right.$ $\left.R_{h e}\right) e^{2} / h$ [51], where the total electron-electron $\left(R_{e e}\right)$ and electron-hole $\left(R_{h e}\right)$ reflection probabilities of free electrons incident on the contact are evaluated at energy $\epsilon=e V$. The full $R$ matrix, including both electron and hole sectors, may be obtained in terms of Caroli's formula $R=\operatorname{Tr}\left(G_{r} \Gamma G_{a} \Gamma\right.$ ), where $G_{r / a}$ are the (dressed) retarded/advanced Green functions in the sample, and $\Gamma=-i\left(\Sigma-\Sigma^{\dagger}\right)$ is (twice) the decay-rate matrix into the normal probe. All these operators are defined in the Nambu basis, just like, e.g., Eq. (D5). The Green's function matrices where solved by inverting the Dyson equation $\left(\epsilon \pm i 0^{+}-H-\Sigma\right) G_{r / a}(\epsilon)=1$ using efficient linear algebra routines, where $H$ is the Nambu Hamiltonian of graphene, including the superconductors. The self-energy $\Sigma=V^{\dagger} g_{r} V$ is defined in terms of the hopping matrix $V$ between graphene and the semi-infinite normal lead, and the retarded Green function $g_{r}$ of the latter. Here, $g_{r}$ is obtained by solving the corresponding (self-consistent) Dyson equation $\left(\epsilon+i 0^{+}-h-v^{\dagger} g_{r} v\right) g_{r}=1$, where $h$ and $v$ are the on-site and hopping matrices acting on the lead's constituent unit cells.

\section{Estimates for canting angles $\boldsymbol{\theta}_{\boldsymbol{L}, W}$}

The canting angle $\theta_{L}$ is defined as the $\theta$ such that the corresponding decay length of edge states along a vacuum edge equals the length $L$ of the Josephson junction [see Fig. 3(a)]. Likewise, $\theta_{W}$ is defined as the $\theta$ such that the corresponding decay length of edge states along a superconducting edge equals the width $W$ (hence, $\theta_{W} \leq \theta_{\text {ins }}$ ).

The evaluation of $\theta_{W}$ can be made by extracting the decay length $1 / \operatorname{Im} k_{x}$ of gap states at zero energy from the effective Hamiltonian of Eq. (B1) without disorder $(w=0)$, and equating that to $W$. The result comes out simply as

$$
\cos \theta_{W} \approx \cos \theta_{\mathrm{ins}}+\frac{2 v_{1} v_{2}}{W^{2} \Delta_{\mathrm{SC}}^{* 2}}
$$

where, recall, $\hbar=1$ and

$$
\cos \left(\theta_{\text {ins }} / 2\right)=\frac{1}{2}\left|\mu_{1} \sqrt{v_{2} / v_{1}}+\mu_{2} \sqrt{v_{1} / v_{2}}\right| / \Delta_{\text {SC }}^{*} .
$$

Note that in the limit $W \rightarrow \infty, \theta_{W}=\theta_{\text {ins }}$, as expected.

An analogous calculation can be done for the vacuum edge along the $y$ direction, whose effective (normal) Hamiltonian can be written in analogy to Eq. (B1) as

$$
H_{\mathrm{eff}}^{\mathrm{vac}} \approx\left(\begin{array}{cc}
\mu_{N}+v_{F} k_{y} & \frac{1}{2} \Delta_{\mathrm{ZLL}} \sin (\theta / 2) \\
\frac{1}{2} \Delta_{\mathrm{ZLL}} \sin (\theta / 2) & \mu_{N}-v_{F} k_{y}
\end{array}\right) .
$$

This leads to the estimate

$$
\sin ^{2} \frac{\theta_{L}}{2} \approx\left(2 \frac{\mu_{N}}{\Delta_{\mathrm{ZLL}}}\right)^{2}+\left(\frac{2 v_{F}}{L \Delta_{\mathrm{ZLL}}}\right)^{2} .
$$

Note that as $L \rightarrow \infty, \theta_{L}$ reaches a minimum value that corresponds to the threshold where the vacuum edge becomes gapped (nonzero for $\mu_{N} \neq 0$ ).

Mean-field results within the Hubbard model (see Appendix A and Ref. [31]) yield a dependence of canting angle $\theta$ with in-plane magnetic field $B_{\|}$of the form $\sin (\theta / 2) \approx \sqrt{1-\left(B_{\|} / B_{0}\right)^{2}}$, where $B_{0}$ is the in-plane field that achieves complete ferromagnetic polarization.

\section{Differential conductance with a single superconducting contact}

The formation and detection of graphene-based Majoranas only requires a single superconducting contact. 
In that sense, the geometry discussed in Fig. 3 of the main text, while relevant in the context of the Josephson effect, Fraunhofer patterns, and parity crossings, is not minimal. A simpler geometry with a single superconducting contact allows for the detection of two Majoranas (instead of four) in the form of a zero-bias anomaly, analogous to that of Fig. 3(c). In Fig. 4, we present the $d I / d V$ in such a geometry. Panel (b) shows the formation of a zero-bias $2 e^{2} / h$ anomaly within $\theta_{W}<\theta<\theta_{L^{*}}$ (with an $L^{*}=2 L+W$ that now corresponds to the total length of the vacuum edge). Note that, while this setup [panel (a)] does not allow for a $\phi$-controlled modulation, its $\theta$ dependence is qualitatively the same as for a Josephson junction. Interestingly, moreover, the $d I / d V$ dependence with the flux $B_{z}$ is very similar to its $\Phi$ dependence in a Josephson junction. The reason is that the flux changes the relative phase of the two Majoranas in the SC contact, making them cross at zero energy each time the flux $\Phi$ is increased by $\Phi_{0}$. This is shown in Fig. 4(c).

\section{Relation of the graphene $d I / d V$ to junctions of topological nanowires}

The transport spectroscopy results for Majoranas in graphene presented in the main text exhibit three different regimes, labeled as $\mathrm{A}, \mathrm{B}$, and $\mathrm{C}$ in Figs. 3 and 4. These regimes arise from the interplay, as a function of canting angle $\theta$, between delocalization of Majorana bound states along either a vacuum edge or the superconducting contact. From the point of view of the normal point contact, the former case (A) is analogous to a one-dimensional TS/ normal/TS Josephson junction, where the normal probe is an extra lead coupled to the normal section for spectrocopy. In contrast, in the case for which the Majoranas remain bound to the corners of the sample and do not delocalize (B), the probe is tunnel coupled to the closest Majorana bound state (top-left corner of the sample) and plays the role of a tunnel normal/TS junction like in the zero-bias anomaly experiment of Ref. [1]. Finally, case C is like case $\mathrm{B}$ albeit for a trivial normal/superconductor junction without Majorana bound states. These mappings to wellunderstood systems are useful to understand the universal values of the $d I / d V$ obtained in each case.

For the case analogous to B, a one-dimensional normal/ TS junction, it is well known [52-58] that the $d I / d V$ for a long enough TS yields a universal $2 e^{2} / h$ zero-bias conductance resonance, a telltale signal of the presence of a Majorana bound state at the contact. If the TS has a finite length, the overlap of the Majorana with its sibling at the opposite end of the TS section gives rise to a splitting of the zero-bias resonance by an energy that is exponentially small in the TS length divided by the spin-orbit length (or, more precisely, the Majorana localization length [59]). A simple model for such an normal/TS junction was devised by Oreg et al. and by Lutchyn et al. in Refs. [16,17]. The model is based on semiconducting wires that exhibit a TS phase when proximized to an $s$ wave $\mathrm{SC}$ while under a longitudinal Zeeman $V_{Z}$ exceeding a critical value $V_{Z}^{c}=\sqrt{\mu^{N}+\Delta^{2}}$. The typical $d I / d V$ in

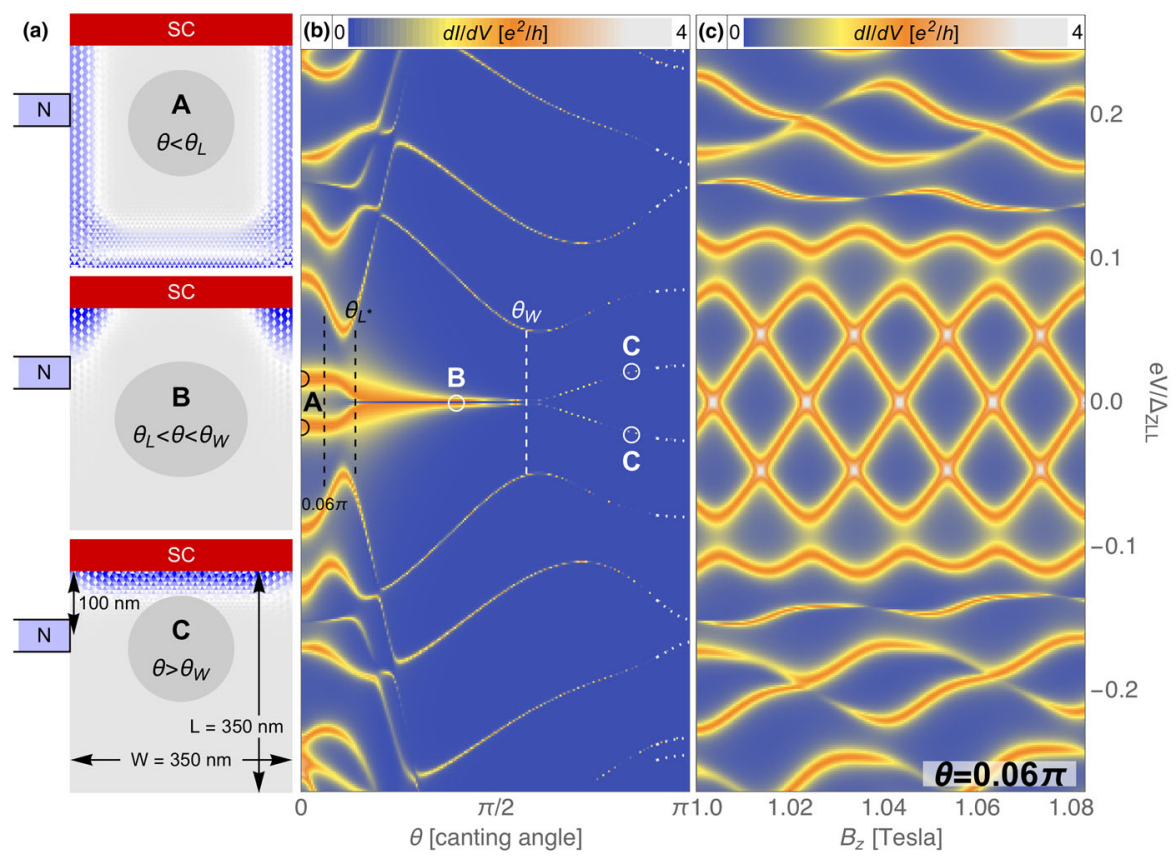

FIG. 4. (a) $350 \mathrm{~nm} \times 350 \mathrm{~nm}$ sample with a single superconducting contact, otherwise identical to system of Fig. 3, main text. (b) and (c) show the differential conductance as a function of canting angle $\theta$ and out of plane magnetic field $B_{z}$. Both show the same phenomenology as panels (b) and (e) of the two contact setup in Fig. 3, albeit with a simpler two-Majorana geometry, and with the total length of the vacuum edge $L^{*}=2 L+W$ playing the role of $L$. 
such a junction as a function of $V_{Z}$ and bias $V$ is shown in Fig. 5(c). Note that, indeed, for $V_{Z}>V_{Z}^{c}$, a zero-bias anomaly of magnitude $2 e^{2} / h$ develops, with a small splitting (oscillating in $V_{Z}$ ) [60-63].

In the case analogous to $\mathrm{A}$, we have a TS/normal/TS, where the normal portion represents the graphene vacuum edge over which the Majoranas delocalize, probed with an additional point contact. The Oreg-Lutchyn model in the topological phase yields a transport spectroscopy map, shown in Fig. 5(b), with a single zero-energy crossing as the junction phase difference $\phi$ increases by $2 \pi$, just like in Figs. 3(b) and 3(d), by virtue of the nontrivial junction topology. Moreover, the zero-bias $d I / d V$ at the crossing is pinned to $4 e^{2} / h$ (white), again like in the graphene case in Figs. 3(b) and 3(d). This universal value can be understood intuitively as the addition in parallel of two $2 e^{2} / h$ normal/ TS zero-bias anomalies, one per Majorana (both are coupled to the probe in this geometry), when the two become decoupled at the appropriate $\phi$.

Finally, note that the trivial SC/normal/SC Josephson junction, corresponding to case $\mathrm{C}$, does not yield a universal zero-bias anomaly at any phase $\phi$ [see Fig. 5(a)]. Instead, the finite-energy Andreev levels yield a $d I / d V$ at finite bias that approaches (nonuniversal) $4 e^{2} / h$. Unlike for the topological case A above, however, perturbations to the system may introduce an additional normal-reflection component to said Andreev levels that suppress this value.

\section{APPENDIX D: HELICAL EDGE STATES IN AN AF GRAPHENE/SUPERCONDUCTING INTERFACE}

\section{Interface states without Landau levels}

The interface states between a superconductor and an antiferromagnetic honeycomb lattice are not related to the Landau-level structure. In the particular case of graphene, the magnetic field is the key ingredient to develop magnetic order (due to the large kinetic energy of electrons), which is developed when the kinetic energy is quenched by the magnetic field. However, in a general honeycomb lattice, provided the interactions are large enough at $B=0$, electrons might be able to develop $A F$ order and thus create interface states at a superconductor contact even at $B=0$. This behavior might be relevant for other honeycomb systems with smaller hopping strengths than graphene, such as silicene, germanene, stanene, or honeycomb oxides.

We show in Fig. 6 that such an interface sustains the same kind of states as in the case of the antiferromagnetic quantum Hall state. In the case of zigzag interfaces [Figs. 6(c) and 6(d)], each valley supports its own set of interface states, whereas for an armchair interface
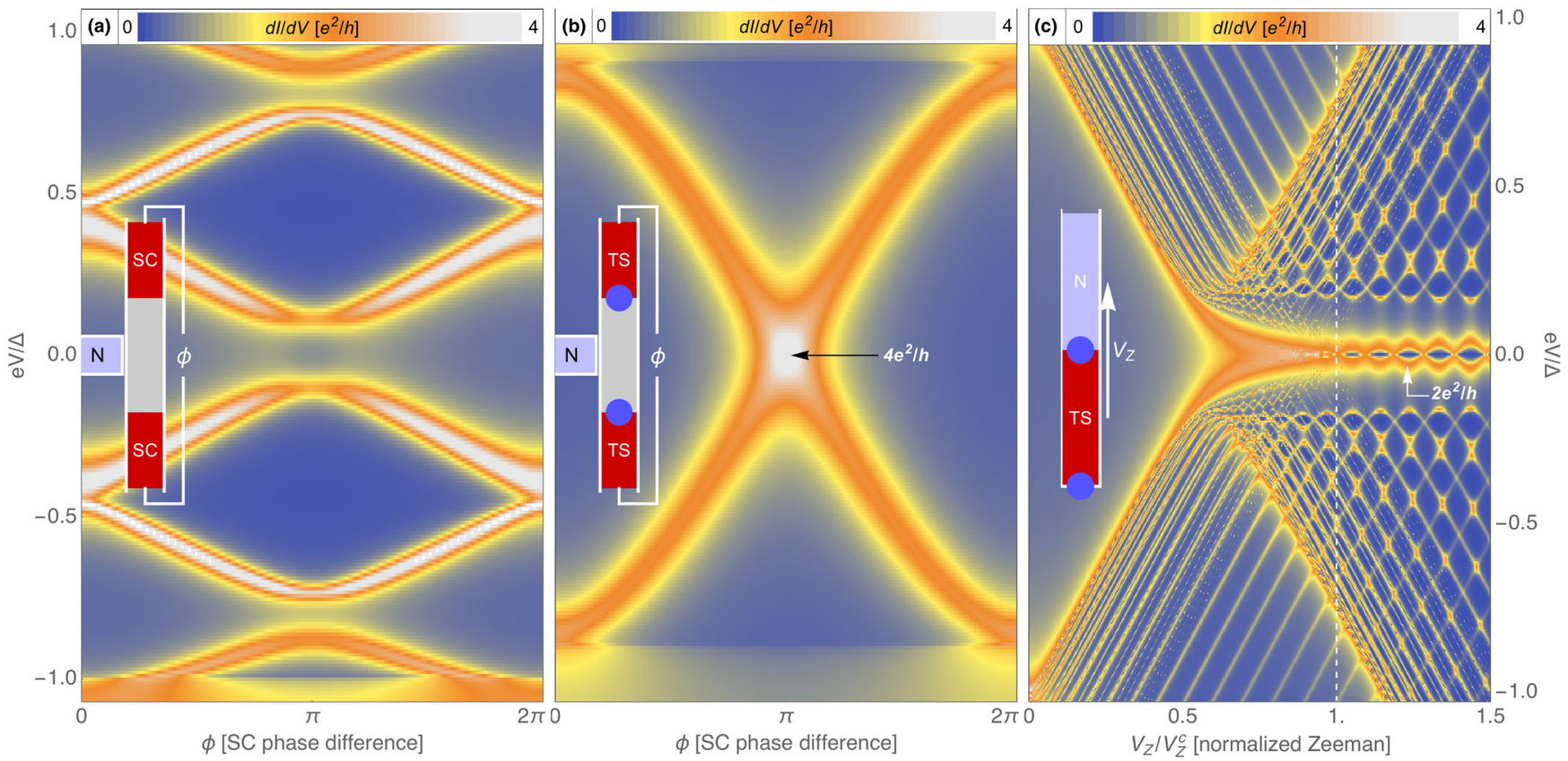

FIG. 5. Transport spectroscopy $d I / d V$ obtained in various arrangements of normal, trivial superconducting (SC), and topological superconducting (TS) nanowires, to be compared to Fig. 3. The wires are modeled after Refs. [16,17]. (a) A trivial SC/normal/SC Josephson junction is probed by a third normal contact at bias $V$, as a function of the superconducting phase difference $\phi$. No protected zero-bias $d I / d V$ anomalies arise. (b) Same as (a) for a TS/normal/TS junction. A universal $4 e^{2} / h$ zero-bias anomaly (white) is obtained at a certain $\phi$, here $\phi=\pi$, for which the two Majoranas in the junction (blue circles in the inset) decouple. (c) A normal/SC junction that transitions into normal/TS with two Majoranas as the longitudinal Zeeman field $V_{Z}$ exceeds a critical value $V_{Z}^{c}$. Apart from a small oscillatory splitting that decays exponentially with TS length, the $d I / d V$ in the latter case shows a universal $2 e^{2} / h$ zero-bias anomaly (orange). 
(a)
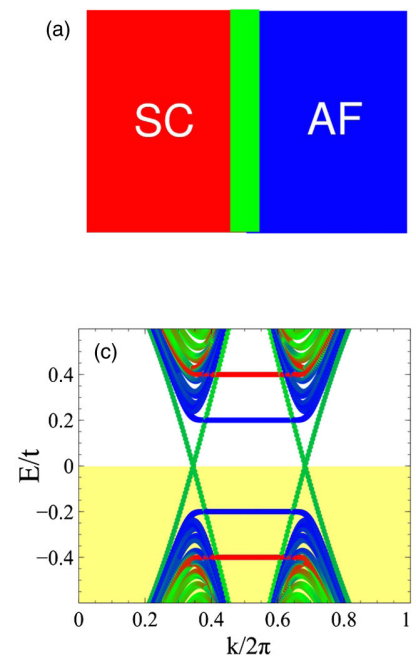
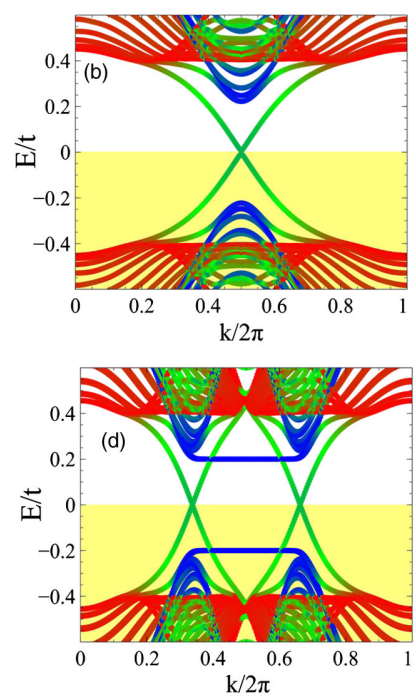

FIG. 6. (a) Sketch of a hybrid AF-SC ribbon, with region colors as those used in the band structure. (b) Band structure of a hybrid ribbon with doped SC and armchair interface. Band structure of a hybrid ribbon with undoped (c) and doped (d) SC with zigzag interface, which shows the different interface states in each valley.

[Fig. 6(b)], the two valleys are folded and interface states between different valleys can couple. In the former case, if the interface is abrupt enough, a small gap opens up because of intervalley mixing.

If a canting in the magnetic moments is introduced, the zigzag interphase remains gapless. The same happens when only an orbital magnetic field is introduced. Only when both perturbations are present simultaneously is the system able to enter into the topological superconducting state. Thus, an off-plane magnetic field is mandatory to observe the Majorana bound states. The previous phenomenology suggests that in order to develop a topological gap, both the spin rotation symmetry and the spatial gauge symmetries have to be broken.

\section{Helical edge states from wave matching}

The interface states between a honeycomb antiferromagnet and a superconductor are not intrinsically related to the Landau-level spectrum. Although in graphene, the antiferromagnetic state is only expected to arise when the system enters the quantum Hall regime, a general antiferromagnetic honeycomb lattice might also sustain interface states when attached to a superconductor without a magnetic flux.

In this section, we show how interface states naturally arise by an analytic argument in the absence of a magnetic field. In particular, a simple wave matching between an $E=0$ energy state shows that the boundary between an antiferromagnet and an $s$-wave superconductor is able to sustain such a state.

To proceed, we look for bounded solutions such that $H|\phi\rangle=0$ with

$$
\begin{gathered}
\phi(x)=\left(\begin{array}{l}
c_{1} \\
c_{2} \\
c_{3} \\
c_{4}
\end{array}\right) r(x)=\left(\begin{array}{l}
c_{1} \\
c_{2} \\
c_{3} \\
c_{4}
\end{array}\right) e^{-\lambda(x) x}, \\
\lambda(x)= \begin{cases}-\Delta_{\mathrm{SC}} & \text { if } x<0 \\
m & \text { if } x>0 .\end{cases}
\end{gathered}
$$

In the following, we focus on one of the four decoupled sectors, in particular, the $|e, \uparrow, K\rangle$ with $\left|h, \downarrow, K^{\prime}\right\rangle$ sector.

For the antiferromagnet, the Hamiltonian reads

$$
H_{A F}=\left(\begin{array}{cccc}
m & p & 0 & 0 \\
p & -m & 0 & 0 \\
0 & 0 & m & -p \\
0 & 0 & -p & -m
\end{array}\right)
$$

whose $E=0$ solutions are

$$
\Phi_{1}=\frac{1}{\sqrt{2}}\left(\begin{array}{c}
1 \\
i \\
0 \\
0
\end{array}\right), \quad \Phi_{2}=\frac{1}{\sqrt{2}}\left(\begin{array}{c}
0 \\
0 \\
1 \\
-i
\end{array}\right) .
$$

On the other hand, for the superconductor, the Hamiltonian reads

$$
H_{S C}=\left(\begin{array}{cccc}
0 & p & \Delta_{\mathrm{SC}} & 0 \\
p & 0 & 0 & \Delta_{\mathrm{SC}} \\
\Delta_{\mathrm{SC}} & 0 & 0 & -p \\
0 & \Delta_{\mathrm{SC}} & -p & 0
\end{array}\right)
$$

with $E=0$ solutions

$$
\Psi_{1}=\frac{1}{\sqrt{2}}\left(\begin{array}{c}
0 \\
1 \\
i \\
0
\end{array}\right), \quad \Psi_{2}=\frac{1}{\sqrt{2}}\left(\begin{array}{l}
1 \\
0 \\
0 \\
i
\end{array}\right)
$$

Imposing continuity at the interface $x=0$, the full $E=0$ solution reads

$$
\begin{aligned}
& \phi(x)=\frac{1}{2}\left(\begin{array}{c}
1 \\
i \\
-1 \\
i
\end{array}\right) r(x), \\
& r(x)= \begin{cases}e^{\Delta_{\mathrm{SC}}} & \text { if } x<0 \\
e^{-m x} & \text { if } x>0,\end{cases}
\end{aligned}
$$


so that a normalizable $E=0$ exists for an interface between a trivial antiferromagnet and a trivial Dirac superconductor.

\section{Helical modes from explicit integration}

In the previous section, we build the $E=0$ state by wave matching across a sharp interface. However, it is possible to give a general solution for the interface state between the antiferromagnet and the superconductor. Without loss of generality, in the following, we assume $m>0$ and $\Delta_{\text {SC }}>0$. The Hamiltonian for an arbitrary antiferromagnet and pairing profile for $k_{y}=0$ reads

$$
H=\gamma_{1} m(x)+\gamma_{2} p+\gamma_{3} \Delta_{\mathrm{SC}}(x),
$$

with $\gamma_{1}, \gamma_{2}, \gamma_{3}$ defined by

$$
\begin{aligned}
& \gamma_{1}=\left(\begin{array}{cccc}
1 & 0 & 0 & 0 \\
0 & -1 & 0 & 0 \\
0 & 0 & 1 & 0 \\
0 & 0 & 0 & -1
\end{array}\right), \\
& \gamma_{2}=\left(\begin{array}{cccc}
0 & 1 & 0 & 0 \\
1 & 0 & 0 & 0 \\
0 & 0 & 0 & -1 \\
0 & 0 & -1 & 0
\end{array}\right), \\
& \gamma_{3}=\left(\begin{array}{llll}
0 & 0 & 1 & 0 \\
0 & 0 & 0 & 1 \\
1 & 0 & 0 & 0 \\
0 & 1 & 0 & 0
\end{array}\right) .
\end{aligned}
$$

Defining $\gamma_{4}=-i \gamma_{2} \gamma_{1}$ and $\gamma_{5}=i \gamma_{2} \gamma_{3}$, the zero-energy equation reads

$$
\left[p+i \gamma_{4} m(x)-i \gamma_{5} \Delta_{\mathrm{SC}}(x)\right] \phi=0 .
$$

The spinor wave function

$$
\phi_{0}=\frac{1}{2}\left(\begin{array}{c}
1 \\
i \\
-1 \\
i
\end{array}\right)
$$

verifies $\gamma_{4} \phi_{0}=\gamma_{5} \phi_{0}=\phi_{0}$, which allows us to build the $E=0$ solution

$$
\phi(x)=N e^{-\int_{0}^{x}\left[m\left(x^{\prime}\right)-\Delta_{\mathrm{SC}}\left(x^{\prime}\right)\right] d x^{\prime}} \phi_{0}
$$

which is normalizable provided that $m(+\infty)>\Delta_{\mathrm{SC}}(+\infty)$ and $\Delta_{\mathrm{SC}}(-\infty)>m(-\infty)$, which is the condition of the domain wall between the superconductor and the antiferromagnet. For the case of step profiles, the solution obtained by wave matching is recovered.

\section{Influence of $\boldsymbol{\mu}_{\mathrm{SC}}$ in the critical Zeeman field}

In the idealized situation in which the superconductor is described as a single-orbital honeycomb lattice at half filling, an arbitrarily small Zeeman field is capable of opening the interface topological gap. However, charge transfer processes are expected to shift the chemical potential of the proximized graphene (SC region in Fig. 1). In this situation, band bending of the interfacial states leads to a one-dimensional gapless state [see Fig. 7(c)]. In order to reach the interfacial topological superconducting state, the bent bands [Fig. 7(c)] have to be moved up in energy. This can be achieved by increasing the in-plane field so that $\theta<\theta_{\text {ins. }}$. The critical in-plane field $B_{x}$ as a function of doping, which separates the gapless and topologically gapped states, is shown in Figs. 7(e) and 7(f). For small $\mu_{\mathrm{SC}}$, the critical field increases linearly, leading to small critical fields at small doping in the SC, whereas for large doping, the critical field is saturated.
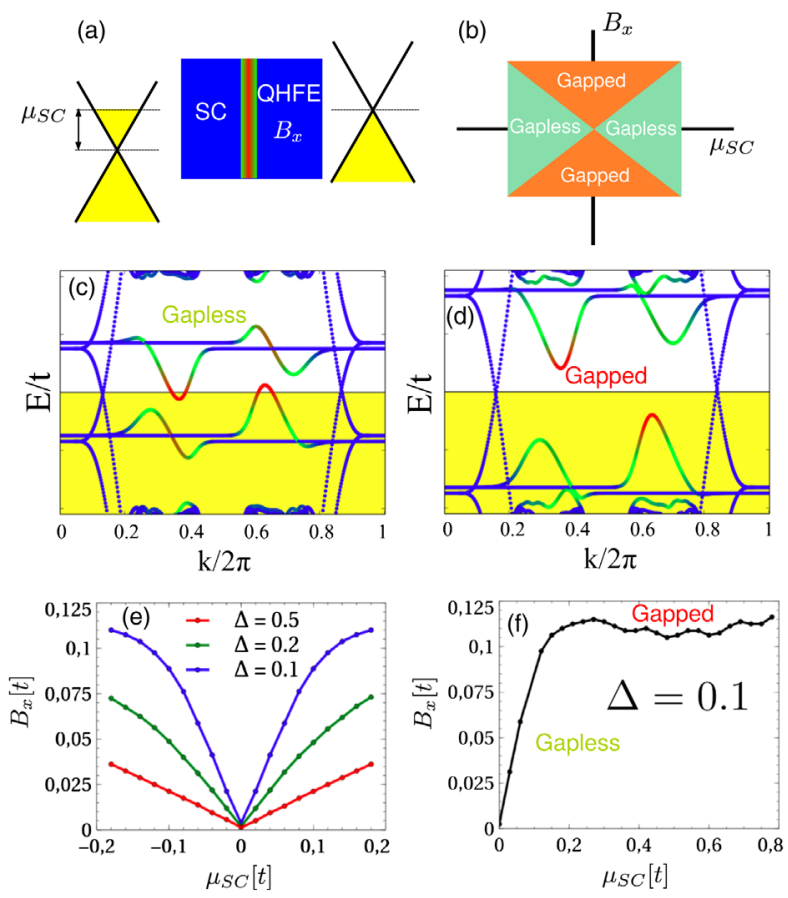

FIG. 7. (a) Scheme of the effect of chemical doping in the Dirac spectrum of proximized graphene. (b) Schematic phase diagram of the interface electronic spectrum between the $\mathrm{SC}$ and the $\mathrm{QH}$ ferromagnet, as a function of the in-plane field and the chemical doping of the SC. Quasiparticle energies for a gapless (c) and gapped (d) interface. Phase boundary obtained by numerical calculation at low dopings (e) and at large doping (f). The green and red colored states of the band structures correspond to the states localized at the interface, where the topological superconducting gap is calculated between the red states shown in $(\mathrm{c}, \mathrm{d})$. The blue gapless states correspond to the chiral states between the $\mathrm{QH}$ and vacuum. 


\section{Emergence of AF helical states from a topological point of view}

An analysis of the emergent AF helical edge modes in terms of topology can be made, but it is less rigorous mathematically than the topological superconductor order in the canted AF phase. As shown in Fig. 1(k), there is a finite volume in parameter space for which the SC contacts are helical metals. The relevant symmetry class within the tenfold way [64] for the infinite system is the 2D class D, and its topological invariant is $\mathbb{Z}$, which corresponds to the number of chiral edge states at a surface [65]. Within this language, the helical SC contact has a trivial (zero) $\mathbb{Z}$ invariant since the number of right- minus left-propagating modes is zero. This is actually the reason why crossing the $\theta=\theta_{\text {ins }}$ destroys the helical edge states without an intervening bulk-gap inversion. Therefore, the reason for the existence of helical states for $\theta>\theta_{\text {ins }}$ cannot be found in the standard homotopy classification. It is rather an instance of a nontrivial valley Chern number.

If one computes the $\mathbb{Z}$ invariant in $2 \mathrm{D}$ in our system, both on the graphene side and on the superconductor side, one needs to integrate the Berry curvature of the Nambu bands. For the superconductor, one obtains negligible Berry curvature for all momenta. However, on the graphene side (and choosing the magnetic unit cell to compute the bands), one finds that while the integrated curvature is zero (hence $\mathbb{Z}$ is zero), it is the sum of two integer and opposite contributions from different valleys. For a specific spin sector (e.g., $|e \uparrow\rangle,|h \downarrow\rangle)$ ), one valley has partial integral 1 and the other -1 . Therefore, assuming valley symmetry is preserved at the graphene-SC interface [that is, $w=0$ in the phase diagram of Fig. 1(k); i.e., the contact is transparent], one can invoke a bulk-boundary correspondence principle for each of the two valleys and spin sectors independently, which yields two pairs of counterpropagating (helical) states (one per valley and spin). If the contact is not transparent $(w>0)$ but intervalley scattering is below the threshold $w<w_{\text {ins }}$, the helical states will split, but the contact will still be metallic (since the splitting is smaller than the energy where they cross). Hence, the AF helical states are an instance of weak topology from the two valleys, just like, e.g., the helical modes in works like Refs. [66,67]. Note, however, that the mathematical standing of these arguments is less sound than the conventional ones from full-Brillouin-zone invariants since, to our knowledge, there is no rigorous theorem that guarantees the existence of surface states from partially integrated (valley) Chern numbers.

\section{APPENDIX E: SQUARE-LATTICE SUPERCONDUCTOR}

In Appendix D, we have considered the superconductor arising from electrons hopping in a honeycomb lattice and subjected to a pairing potential. Nevertheless, the fact that
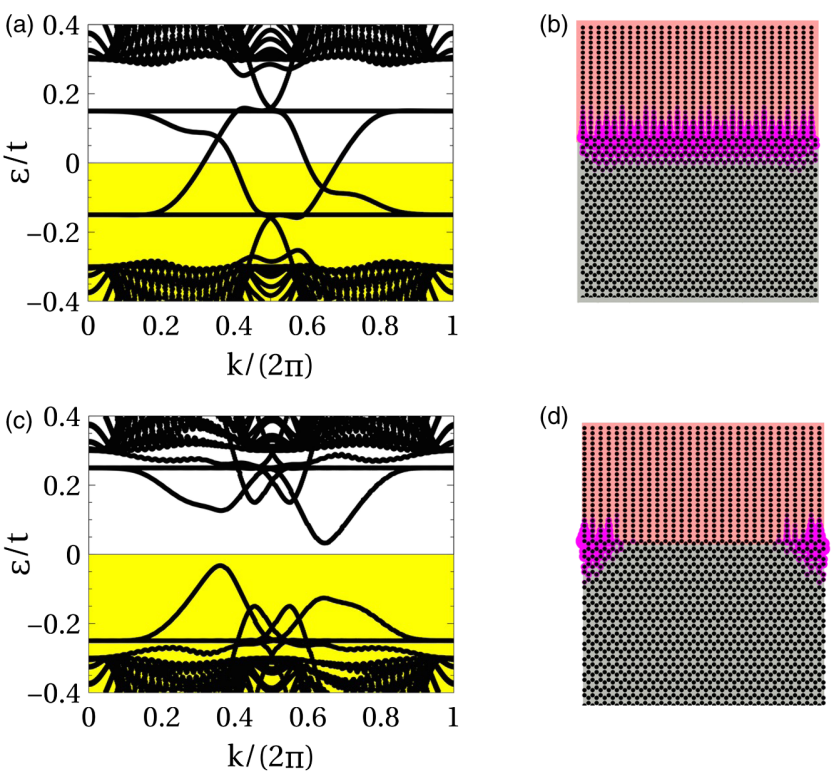

FIG. 8. Band structure of the interface between a square superconductor and an antiferromagnet (a) or canted antiferromagnet (c) honeycomb lattice. Panels (b) and (d) show the local density of states in a finite system, which correspond to gapless interface channels (without canting) (b) and Majorana states (with canting) (d).

the interface states persist even upon doping of the superconductor suggests that their existence goes far beyond what our analytic argument might suggest. Actually, we show here that a honeycomb superconducting lattice is not mandatory, so even an interface between a square superconductor will give rise to localized Majorana states.

To illustrate this, we show in Fig. 8 the band structure of an interface between the canted quantum Hall antiferromagnet and the local density of states for a finite system. In the case of fully collinear antiferromagnetism, the interface sustains a gapless channel. When the moments are canted, a topological gap in the interfacial bands opens up and localized Majorana modes appear.

[1] V. Mourik, K. Zuo, S. M. Frolov, S. R. Plissard, E. P. A. M. Bakkers, and L. P. Kouwenhoven, Signatures of Majorana Fermions in Hybrid Superconductor-Semiconductor Nanowire Devices, Science 336, 1003 (2012).

[2] M. T. Deng, C. L. Yu, G. Y. Huang, M. Larsson, P. Caroff, and H. Q. Xu, Anomalous Zero-Bias Conductance Peak in a Nb-InSb Nanowire-Nb Hybrid Device, Nano Lett. 12, 6414 (2012).

[3] A. Das, Y. Ronen, Y. Most, Y. Oreg, M. Heiblum, and H. Shtrikman, Zero-Bias Peaks and Splitting in an Al-InAs Nanowire Topological Superconductor as a Signature of Majorana Fermions, Nat. Phys. 8, 887 (2012).

[4] L. P. Rokhinson, X. Liu, and J. K. Furdyna, The Fractional a.c. Josephson Effect in a Semiconductor-Superconductor 
Nanowire as a Signature of Majorana Particles, Nat. Phys. 8, 795 (2012).

[5] A. D. K. Finck, D. J. Van Harlingen, P. K. Mohseni, K. Jung, and X. Li, Anomalous Modulation of a Zero-Bias Peak in a Hybrid Nanowire-Superconductor Device, Phys. Rev. Lett. 110, 126406 (2013).

[6] H. O. H. Churchill, V. Fatemi, K. Grove-Rasmussen, M. T. Deng, P. Caroff, H. Q. Xu, and C. M. Marcus, Superconductor-Nanowire Devices from Tunneling to the Multichannel Regime: Zero-Bias Oscillations and Magnetoconductance Crossover, Phys. Rev. B 87, 241401 (2013).

[7] E. J. H. Lee, X. Jiang, M. Houzet, R. Aguado, C. M. Lieber, and S. De Franceschi, Spin-Resolved Andreev Levels and Parity Crossings in Hybrid Superconductor-Semiconductor Nanostructures, Nat. Nanotechnol. 9, 79 (2014).

[8] S. Hart, H. Ren, T. Wagner, P. Leubner, M. Muhlbauer, C. Brune, H. Buhmann, L. W. Molenkamp, and A. Yacoby, Induced Superconductivity in the Quantum Spin Hall Edge, Nat. Phys. 10, 638 (2014).

[9] V.S. Pribiag, A. J. A. Beukman, F. Qu, M. C. Cassidy, C. Charpentier, W. Wegscheider, and L. P. Kouwenhoven, Edge-Mode Superconductivity in a Two-Dimensional Topological Insulator, Nat. Nanotechnol. 10, 593 (2015).

[10] S. Nadj-Perge, I. K. Drozdov, J. Li, H. Chen, S. Jeon, J. Seo, A. H. MacDonald, B. A. Bernevig, and A. Yazdani, Observation of Majorana Fermions in Ferromagnetic Atomic Chains on a Superconductor, Science 346, 602 (2014).

[11] R. Pawlak, M. Kisiel, J. Klinovaja, T. Meier, S. Kawai, T. Glatzel, D. Loss, and E. Meyer, Probing Atomic Structure and Majorana Wavefunctions in Mono-atomic Fe-Chains on Superconducting Pb-Surface, arXiv:1505.06078.

[12] C. Kurter, A. D. K. Finck, Y.S. Hor, and D. J. Van Harlingen, Evidence for an Anomalous Current-Phase Relation in Topological Insulator Josephson Junctions, Nat. Commun. 6, 7130 (2015).

[13] J. Wiedenmann, E. Bocquillon, R. S. Deacon, S. Hartinger, T. M. Klapwijk, L. Maier, C. Ames, C. Brüne, K. Ishibashi, S. Tarucha, H. Buhmann, and L. W. Molenkamp, ZeroEnergy Andreev Bound States in a HgTe-Based Topological Josephson Junction, arXiv:1503.05591.

[14] L. Fu and C. L. Kane, Superconducting Proximity Effect and Majorana Fermions at the Surface of a Topological Insulator, Phys. Rev. Lett. 100, 096407 (2008).

[15] J. D. Sau, R. M. Lutchyn, S. Tewari, and S. Das Sarma, Generic New Platform for Topological Quantum Computation Using Semiconductor Heterostructures, Phys. Rev. Lett. 104, 040502 (2010).

[16] R. M. Lutchyn, J. D. Sau, and S. Das Sarma, Majorana Fermions and a Topological Phase Transition in Semiconductor-Superconductor Heterostructures, Phys. Rev. Lett. 105, 077001 (2010).

[17] Y. Oreg, G. Refael, and F. von Oppen, Helical Liquids and Majorana Bound States in Quantum Wires, Phys. Rev. Lett. 105, 177002 (2010).

[18] A. F. Young, C. R. Dean, L. Wang, H. Ren, P. CaddenZimansky, K. Watanabe, T. Taniguchi, J. Hone, K. L. Shepard, and P. Kim, Spin and Valley Quantum Hall Ferromagnetism in Graphene, Nat. Phys. 8, 550 (2012).
[19] A. F. Young, J. D. Sanchez-Yamagishi, B. Hunt, S. H. Choi, K. Watanabe, T. Taniguchi, R. C. Ashoori, and P. JarilloHerrero, Tunable Symmetry Breaking and Helical Edge Transport in a Graphene Quantum Spin Hall State, Nature (London) 505, 528 (2014).

[20] V. E. Calado, S. Goswami, G. Nanda, M. Diez, A. R. Akhmerov, K. Watanabe, T. Taniguchi, T. M. Klapwijk, and L. M. K. Vandersypen, Ballistic Josephson Junctions in Edge-Contacted Graphene, Nat. Nanotechnol. 10, 761 (2015).

[21] M. Ben Shalom, M. J. Zhu, V. I. Fal'ko, A. Mishchenko, A. V. Kretinin, K. S. Novoselov, C. R. Woods, K. Watanabe, T. Taniguchi, A. K. Geim, and J. R. Prance, Proximity Superconductivity in Ballistic Graphene, from Fabry-Perot Oscillations to Random Andreev States in Magnetic Field, arXiv:1504.03286.

[22] A. H. C. Neto, F. Guinea, N. M. R. Peres, K. S. Novoselov, and A. K. Geim, The Electronic Properties of Graphene, Rev. Mod. Phys. 81, 109 (2009).

[23] C. L. Kane and E. J. Mele, Quantum Spin Hall Effect in Graphene, Phys. Rev. Lett. 95, 226801 (2005).

[24] Y. Zhang, Z. Jiang, J. P. Small, M. S. Purewal, Y.-W. Tan, M. Fazlollahi, J. D. Chudow, J. A. Jaszczak, H. L. Stormer, and P. Kim, Landau-Level Splitting in Graphene in High Magnetic Fields, Phys. Rev. Lett. 96, 136806 (2006).

[25] K. Bolotin, F. Ghahari, M. Shulman, H. Stormer, and P. Kim, Observation of the Fractional Quantum Hall Effect in Graphene, Nature (London) 462, 196 (2009).

[26] D. L. Miller, K. D. Kubista, G. M. Rutter, M. Ruan, W. A. de Heer, P. N. First, and J. A. Stroscio, Observing the Quantization of Zero Mass Carriers in Graphene, Science 324, 924 (2009).

[27] B. E. Feldman, B. Krauss, J. H. Smet, and A. Yacoby, Unconventional Sequence of Fractional Quantum Hall States in Suspended Graphene, Science 337, 1196 (2012).

[28] A. Kou, B. E. Feldman, A. J. Levin, B. I. Halperin, K. Watanabe, T. Taniguchi, and A. Yacoby, Electron-Hole Asymmetric Integer and Fractional Quantum Hall Effect in Bilayer Graphene, Science 345, 55 (2014).

[29] G. L. Yu, R. V. Gorbachev, J. S. Tu, A. V. Kretinin, Y. Cao, R. Jalil, F. Withers, L. A. Ponomarenko, B. A. Piot, M. Potemski, D. C. Elias, X. Chen, K. Watanabe, T. Taniguchi, I. V. Grigorieva, K. S. Novoselov, V. I. Fal'ko, A. K. Geim, and A. Mishchenko, Hierarchy of Hofstadter States and Replica Quantum Hall Ferromagnetism in Graphene Superlattices, Nat. Phys., advance online publication (2014).

[30] I. F. Herbut, Theory of Integer Quantum Hall Effect in Graphene, Phys. Rev. B 75, 165411 (2007).

[31] J. L. Lado and J. Fernández-Rossier, Noncollinear Magnetic Phases and Edge States in Graphene Quantum Hall Bars, Phys. Rev. B 90, 165429 (2014).

[32] M. Kharitonov, Phase Diagram for the $\nu=0$ Quantum Hall State in Monolayer Graphene, Phys. Rev. B 85, 155439 (2012).

[33] Recently, schemes to experimentally probe the AF phase through spin excitations have been proposed [68].

[34] H. A. Fertig and L. Brey, Luttinger Liquid at the Edge of Undoped Graphene in a Strong Magnetic Field, Phys. Rev. Lett. 97, 116805 (2006). 
[35] D. A. Abanin, P. A. Lee, and L. S. Levitov, Spin-Filtered Edge States and Quantum Hall Effect in Graphene, Phys. Rev. Lett. 96, 176803 (2006).

[36] X.-L. Qi and S.-C. Zhang, Topological Insulators and Superconductors, Rev. Mod. Phys. 83, 1057 (2011).

[37] M. Wimmer, A. R. Akhmerov, J. P. Dahlhaus, and C. W. J. Beenakker, Quantum Point Contact as a Probe of a Topological Superconductor, New J. Phys. 13, 053016 (2011).

[38] M. T. Allen, O. Shtanko, I. C. Fulga, A. Akhmerov, K. Watanabi, T. Taniguchi, P. Jarillo-Herrero, L. S. Levitov, and A. Yacoby, Spatially Resolved Edge Currents and GuidedWave Electronic States in Graphene, arXiv:1504.07630.

[39] S.-P. Lee, K. Michaeli, J. Alicea, and A. Yacoby, Revealing Topological Superconductivity in Extended Quantum Spin Hall Josephson Junctions, Phys. Rev. Lett. 113, 197001 (2014).

[40] A. Yu Kitaev, Unpaired Majorana Fermions in Quantum Wires, Phys. Usp. 44, 131 (2001).

[41] T. Dirks, T. L. Hughes, S. Lal, B. Uchoa, Y.-F. Chen, C. Chialvo, P. M. Goldbart, and N. Mason, Transport through Andreev Bound States in a Graphene Quantum Dot, Nat. Phys. 7, 386 (2011).

[42] H. J. Kwon, K. Sengupta, and V. M. Yakovenko, Fractional ac Josephson Effect in $p$ - and $d$-wave Superconductors, Eur. Phys. J. B 37, 349 (2003).

[43] L. Fu and C. L. Kane, Josephson Current and Noise at a Superconductor/Quantum-Spin-Hall-Insulator/Superconductor Junction, Phys. Rev. B 79, 161408 (2009).

[44] P. San-Jose, E. Prada, and R. Aguado, ac Josephson Effect in Finite-Length Nanowire Junctions with Majorana Modes, Phys. Rev. Lett. 108, 257001 (2012).

[45] P. San-Jose, E. Prada, and R. Aguado, Mapping the Topological Phase Diagram of Multiband Semiconductors with Supercurrents, Phys. Rev. Lett. 112, 137001 (2014).

[46] J. Cayao, E. Prada, P. San-Jose, and R. Aguado, SNS Junctions in Nanowires with Spin-Orbit Coupling: Role of Confinement and Helicity on the Subgap Spectrum, Phys. Rev. B 91, 024514 (2015).

[47] D. K. Efetov, L. Wang, C. Handschin, K. B. Efetov, J. Shuang, R. Cava, T. Taniguchi, K. Watanabe, J. Hone, C. R. Dean, and P. Kim, Specular Interband Andreev Reflections in Graphene, arXiv:1505.04812.

[48] M.-H. Liu, P. Rickhaus, P. Makk, E. Tóvári, R. Maurand, F. Tkatschenko, M. Weiss, C. Schönenberger, and K. Richter, Scalable Tight-Binding Model for Graphene, Phys. Rev. Lett. 114, 036601 (2015).

[49] C. W. J. Beenakker, Three Universal Mesoscopic Josephson Effects, in Transport Phenomena in Mesoscopic Systems: Proceedings of the 14th Taniguchi Symposium, Shima, Japan, 1991 (Springer-Verlag, Berlin, 1992).

[50] S. Tewari and J. D. Sau, Topological Invariants for SpinOrbit Coupled Superconductor Nanowires, Phys. Rev. Lett. 109, 150408 (2012).

[51] G. E. Blonder, M. Tinkham, and T. M. Klapwijk, Transition from Metallic to Tunneling Regimes in Superconducting
Microconstrictions: Excess Current, Charge Imbalance, and Supercurrent Conversion, Phys. Rev. B 25, 4515 (1982).

[52] K. T. Law, P. A. Lee, and T. K. Ng, Majorana Fermion Induced Resonant Andreev Reflection, Phys. Rev. Lett. 103, 237001 (2009).

[53] B. Béri, Dephasing-Enabled Triplet Andreev Conductance, Phys. Rev. B 79, 245315 (2009).

[54] K. Flensberg, Tunneling Characteristics of a Chain of Majorana Bound States, Phys. Rev. B 82, 180516 (2010).

[55] L. Fidkowski, J. Alicea, N. Lindner, R. M. Lutchyn, and M. P. A. Fisher, Universal Transport Signatures of Majorana Fermions in Superconductor-Luttinger Liquid Junctions, Phys. Rev. B 85, 245121 (2012).

[56] D. I. Pikulin, J. P. Dahlhaus, M. Wimmer, H. Schomerus, and C. W. J. Beenakker, A Zero-Voltage Conductance Peak from Weak Antilocalization in a Majorana Nanowire, New J. Phys. 14, 125011 (2012).

[57] D. I. Pikulin and Y. V. Nazarov, Two Types of Topological Transitions in Finite Majorana Wires, Phys. Rev. B 87, 235421 (2013).

[58] P. A. Ioselevich and M. V. Feigel'man, Tunneling Conductance Due to a Discrete Spectrum of Andreev States, New J. Phys. 15, 055011 (2013).

[59] J. Klinovaja and D. Loss, Composite Majorana Fermion Wave Functions in Nanowires, Phys. Rev. B 86, 085408 (2012).

[60] E. Prada, P. San-Jose, and R. Aguado, Transport Spectroscopy of ns Nanowire Junctions with Majorana Fermions, Phys. Rev. B 86, 180503(R) (2012).

[61] J. S. Lim, L. Serra, R. López, and R. Aguado, MagneticField Instability of Majorana Modes in Multiband Semiconductor Wires, Phys. Rev. B 86, 121103 (2012).

[62] S. Das Sarma, J. D. Sau, and T. D. Stanescu, Splitting of the Zero-Bias Conductance Peak as Smoking Gun Evidence for the Existence of the Majorana Mode in a SuperconductorSemiconductor Nanowire, Phys. Rev. B 86, 220506 (2012).

[63] D. Rainis, L. Trifunovic, J. Klinovaja, and D. Loss, Towards a Realistic Transport Modeling in a Superconducting Nanowire with Majorana Fermions, Phys. Rev. B 87, 024515 (2013).

[64] A. Altland and M. R. Zirnbauer, Nonstandard Symmetry Classes in Mesoscopic Normal-Superconducting Hybrid Structures, Phys. Rev. B 55, 1142 (1997).

[65] A. P. Schnyder, S. Ryu, A. Furusaki, and A. W. W. Ludwig, Classification of Topological Insulators and Superconductors in Three Spatial Dimensions, Phys. Rev. B 78, 195125 (2008).

[66] I. Martin, Y. M. Blanter, and A. F. Morpurgo, Topological Confinement in Bilayer Graphene, Phys. Rev. Lett. 100, 036804 (2008).

[67] P. San-Jose and E. Prada, Helical Networks in Twisted Bilayer Graphene under Interlayer Bias, Phys. Rev. B 88, 121408 (2013).

[68] S. Takei, A. Yacoby, B. I. Halperin, and Y. Tserkovnyak, Spin Superfluidity in the $\mathrm{OE} \Omega=0$ Quantum Hall State of Graphene, arXiv:1506.01061. 